Service social

\title{
Oser-Quand des femmes passent à l'action, par le collectif d'écriture du Centre Femmes des Cantons, en collaboration avec Henri Lamoureux, Cowansville, La Collective par et pour elle inc., 1987, 121 pages.
}

\section{Jean Panet-Raymond}

Volume 36, numéro 2-3, 1987

Approches intégrées

URI : https://id.erudit.org/iderudit/706377ar

DOI : https://doi.org/10.7202/706377ar

Aller au sommaire du numéro

Éditeur(s)

École de service social de l'Université Laval

ISSN

1708-1734 (numérique)

Découvrir la revue

Citer ce compte rendu

Panet-Raymond, J. (1987). Compte rendu de [Oser-Quand des femmes passent à

l'action, par le collectif d'écriture du Centre Femmes des Cantons, en

collaboration avec Henri Lamoureux, Cowansville, La Collective par et pour elle inc., 1987, 121 pages.] Service social, 36(2-3), 497-499.

https://doi.org/10.7202/706377ar d'utilisation que vous pouvez consulter en ligne.

https://apropos.erudit.org/fr/usagers/politique-dutilisation/ 
L'auteur utilise, pour décrire son approche de groupe, un style clair et précis; son analyse méthodique est étayée de nombreux exemples tirés de son enseignement et de sa pratique, ce qui rend la lecture du volume très agréable.

Ce livre s'adresse spécialement aux praticien(ne)s, enseignant(e)s et étudiant(e)s en service social, mais il servira également aux autres professions qui veulent se donner des outils de travail avec un groupe.

En ce sens, la traduction du volume en français ${ }^{4}$ est une heureuse initiative ; elle permettra aux praticiens du milieu québécois de se familiariser avec l'approche de Ken Heap, dont c'est le premier volume à paraître en français. La traduction transmet assez fidèlement l'idée de l'auteur dans son ensemble; sauf pour l'expression « to fire worker » qui a été rendue par « mettre le travailleur social au feu » - (page 109) - (au feu nourri de la supervision, peut-être ?) plutôt que par "congédier le travailleur social."

Voilà sûrement un ouvrage à recommander à tous ceux qui veulent travailler avec les groupes et se familiariser avec une approche qui rejoint l'essentiel du groupe, c'est-à-dire son processus.

École de service social,

Céline BÉDARD

Université Laval.

\section{Notes et références}

1 Heap, Ken, Group Theory for Social Workers, An Introduction, Oxford, Pergamon Press, 1977.

2 Heap, Ken, Process and Action in Work with Groups, Oxford, Pergamon Press, 1979.

${ }^{3}$ Heap, Ken, The Practice of Social Work with Groups - A Systematic Approach, London, George Allen and Unwin, 1985, 192 pages.

4 La traduction est de Pierrette Brosset, directrice de l'École Normale Sociale de l'Ouest.

Oser - Quand des femmes passent à l'action, par le collectif d'écriture du Centre Femmes des Cantons, en collaboration avec Henri LAMOUREUX, Cowansville, La Collective par et pour elle inc., 1987, 121 pages.

Il est rare que l'on lise un petit livre aussi stimulant et rafraîchissant, résultat d'une écriture collective par des femmes... avec l'aide d'un écrivain de métier, qui est aussi un vieux militant qui a suivi tout le projet de très près. Cette aide venant d'un homme peut paraitre contradictoire de la part d'un collectif féministe. Mais c'est aussi peut-être symptomatique d'un nouveau féminisme 
qui respire la confiancè et l'ouverture. Ne faut-il pas avoir confiance en soi pour s'ouvrir sur les autres. Et ce livre est un témoignage de confiance.

"Nous n'avons pas d'autres prétentions que d'apporter un témoignage, le plus vivant possible d'un cheminement, d'une démarche dans le cadre de laquelle il nous a été permis d'affirmer notre capacité à devenir les sujets de notre propre développement. " (p. 5)

"Notre lutte fut, de l'avis unanime de celles qui y ont participé, une excellente école de confiance en soi." (p. 92)

Ce livre, par son contenu, son orientation idéologique et son style fait un peu la transition entre les années 70 et 80 . Ancré dans un féminisme des années 70 , il montre le style d'action que l'on peut (et doit !) mener dans les années 80 . En fait, ce livre est surtout intéressant pour des intervenantes et intervenants qui veulent comprendre le passage de l'action individuelle à l'action collective, ou de la conscience "naïve " à la conscience "critique"

"Notre lutte contre la pornographie fut aussi un révélateur, un stimulant. Elle nous a permis d'apprendre que nous étions capables de jouer un rôle concret dans le développement de notre collectivité. " (p. 5)

Ce livre illustre donc le processus d'une action communautaire menée par des femmes qui venaient poursuivre une démarche de développement personnel. C'est à travers l'action collective qu'elles se développeront le plus.

"Nous avons aussi appris que nous étions capables de réaliser des activités pour lesquelles nous ne nous sentions pas douées: nous avons bâti des stratégies, organisé des conférences de presse, monté une pièce de théâtre, rédigé un projet de règlement municipal, parlé en public, polémiqué avec ceux et celles qui opposaientl..." (p. 8)

C'est donc par une lutte contre l'une des plus pernicieuses formes de l'oppression des femmes qu'elles ont pris conscience de leur solidarité nécessaire et de leur force. Cette lutte illustre bien comment le féminisme a su s'ancrer dans des préoccupations larges et concrètes, bien au-delà d'un débat strictement féministe.

Le livre présente d'abord la création de la "Collective par et pour elle " suite à une assemblée sur la violence faite aux femmes convoquées par le Service d'éducation aux adultes. La Collective, qui se voulait un lieu d'action militante et d'apprentissage, a ensuite mis sur pied le Centre Femmes des Cantons à Cowansville.

Le chapitre le plus important et sans doute le plus riche pour les intervenantes est celui qui décrit le cheminement du "Comité de lutte contre la pornographie ". On s'est attardé ici à faire le bilan de cette lutte depuis les origines jusqu'à la victoire ultime, une action qui s'est déroulée sur près de deux ans (1983-84). Ce qui fait la richesse de cette lutte, c'est son évolution. Elle était le fait de femmes sans expérience de l'intervention communautaire qui ont appris très vite.

"Portées pour ainsi dire par la dynamique de notre lutte, nous nous sommes rendu compte qu'en fait, ce combat contre l'exploitation nous a permis de découvrir un large éventail de nos capacités et d'explorer des contrées où nous avions peu ou jamais mis les pieds. " (p. 64) 
C'est dans cette partie que l'on voit le plus l'influence du collaborateur Lamoureux (coauteur de L'Intervention communautaire, Montréal, Saint-Martin, 1984) qui décrit les étapes d'une action communautaire : la base du regroupement, l'identification des objectifs et le choix des cibles, la stratégie et les tactiques, les liens avec la population, la négociation et l'art du repli, l'utilisation des médias. On note particulièrement l'importance des médias dans une région semi-rurale.

"Le Comité informa systématiquement les médias des appuis qu'il recevait... Nous avons maintenu un intérêt permanent des médias pour l'ensemble de notre démarche. Dans un milieu comme le nôtre, où les médias régionaux et locaux sont fort importants, cet appui était indispensable. » (p. 76)

On décrit aussi longuement le mode de fonctionnement démocratique du Comité, le rôle des personnes ressources et les niveaux de participation de toutes et chacune. On a même tenu à intégrer des hommes à l'action.

"Pour réaliser cette jonction, nous avons choisi de ne rien faire de spécial pour atteindre les hommes, sinon de ne pas nous engager dans des tactiques de dénonciation globale, style "tous les hommes sont des pornocrates".» (p. 85)

On décrit, après ce long bilan de la lutte, la recherche menée sur les liens entre la violence et la pornographie, financée par le Secrétariat d'État.

Enfin, on termine le cheminement du Centre des femmes en parlant du projet de Centre de préparation à l'emploi mis sur pied au printemps 1986 et de l'intervention en cours sur le transport public en région.

Ainsi, cette œuvre collective se veut un témoignage de femmes qui ont découvert l'action communautaire et politique par l'implication dans le développement d'une région. C'est un message d'espoir.

La lecture est facile et les témoignages de femmes sont nombreux, mais on subit parfois un ton un peu trop didactique voire moralisateur sur l'éthique de l'intervention, les valeurs défendues, l'importance de la démocratie et de la solidarité. C'est sans doute le côté un peu "années 70 " du collaborateur qui ressort à travers une démarche indiscutablement importante et à multiplier dans d'autres régions.

École de service social,

Jean PANET-RAYMOND

Université de Montréal.

La prostitution, une affaire de sens, par Jean-Guy NADEAU, Montréal, Fides, 1987, 469 pages.

Dans la préface de cet ouvrage, Jacques Grand'Maison dit qu' «il y a des livres qui sont plus que des livres tellement ils sont chargés d'humanité, de vérité sur nous-mêmes". L'énoncé paraît taillé sur mesure tant il s'applique bien au livre de Jean-Guy Nadeau. 